\title{
Stereotactic body radiation therapy for spinal metastases: a novel local control stratification by spinal region
}

\author{
Roman O. Kowalchuk, MD, ${ }^{1}$ Michael R. Waters, MD, PhD, ${ }^{1}$ K. Martin Richardson, MS, ${ }^{1}$ \\ Kelly Spencer, MS, ${ }^{1}$ James M. Larner, MD, ${ }^{2}$ William H. McAllister, MD, ${ }^{3}$ \\ Jason P. Sheehan, MD, PhD, ${ }^{4}$ and Charles R. Kersh, MD ${ }^{1}$
}

\begin{abstract}
${ }^{1}$ Radiosurgery Center, Riverside Regional Medical Center (in partnership with University of Virginia Health System), Newport News; Departments of ${ }^{2}$ Radiation Oncology and ${ }^{4}$ Neurosurgery, University of Virginia Health System, Charlottesville; and ${ }^{3}$ Department of Neurosurgery, Riverside Regional Medical Center, Newport News, Virginia
\end{abstract}

\begin{abstract}
OBJECTIVE This study evaluated a large cohort of patients treated with stereotactic body radiation therapy for spinal metastases and investigated predictive factors for local control, local progression-free survival (LPFS), overall survival, and pain response between the different spinal regions.

METHODS The study was undertaken via retrospective review at a single institution. Patients with a tumor metastatic to the spine were included, while patients with benign tumors or primary spinal cord cancers were excluded. Statistical analysis involved univariate analysis, Cox proportional hazards analysis, the Kaplan-Meier method, and machine learning techniques (decision-tree analysis).
\end{abstract}

RESULTS A total of 165 patients with 190 distinct lesions met all inclusion criteria for the study. Lesions were distributed throughout the cervical (19\%), thoracic (43\%), lumbar (19\%), and sacral $(18 \%)$ spines. The most common treatment regimen was $24 \mathrm{~Gy}$ in 3 fractions (44\%). Via the Kaplan-Meier method, the 24 -month local control was $80 \%$. Sacral spine lesions demonstrated decreased local control $(p=0.01)$ and LPFS $(p<0.005)$ compared with those of the thoracolumbar spine. The cervical spine cases had improved local control $(p<0.005)$ and LPFS $(p<0.005)$ compared with the sacral spine and trended toward improvement relative to the thoracolumbar spine. The 36-month local control rates for cervical, thoracolumbar, and sacral tumors were $86 \%, 73 \%$, and $44 \%$, respectively. Comparably, the 36 -month LPFS rates for cervical, thoracolumbar, and sacral tumors were $85 \%, 67 \%$, and $35 \%$, respectively. A planning target volume (PTV) $>50 \mathrm{~cm}^{3}$ was also predictive of local failure $(p=0.04)$. Fewer cervical spine cases had disease with PTV $>50 \mathrm{~cm}^{3}$ than the thoracolumbar $\left(p=5.87 \times 10^{-8}\right)$ and sacral $\left(p=3.9 \times 10^{-3}\right)$ cases. Using decision-tree analysis, the highest-fidelity models for predicting pain-free status and local failure demonstrated the first splits as being cervical and sacral location, respectively.

CONCLUSIONS This study presents a novel risk stratification for local failure and LPFS by spinal region. Patients with metastases to the sacral spine may have decreased local control due to increased PTV, especially with a PTV of $>50$ $\mathrm{cm}^{3}$. Multidisciplinary care should be emphasized in these patients, and both surgical intervention and radiotherapy should be strongly considered.

https://thejns.org/doi/abs/10.3171/2020.6.SPINE20861

KEYWORDS SBRT; stereotactic body radiation therapy; SABR; stereotactic ablative radiosurgery; metastasis; local control; oncology

$\mathrm{S}$ TEREOTACTIC body radiation therapy (SBRT) is a safe and efficacious technique of delivering ablative doses of therapeutic radiation to a highly conformal target. ${ }^{1}$ Also known as stereotactic ablative radiotherapy, one of its most common uses is for spinal metastases. ${ }^{2,3}$ The two chief outcomes generally are local tumor control and pain control; however, wide ranges in the criteria used to quantify pain response have made rigorously studying this benefit difficult. ${ }^{4}$ Even so, encouraging results have been published in phase II trials, including a comparison with

ABBREVIATIONS BED = biologically effective dose; KPS = Karnofsky Performance Status; $\mathrm{LPFS}=$ local progression-free survival; $\mathrm{PTV}=$ planning target volume; SBRT = stereotactic body radiation therapy.

SUBMITTED May 12, 2020. ACCEPTED June 16, 2020.

INCLUDE WHEN CITING Published online October 23, 2020; DOI: 10.3171/2020.6.SPINE20861. 
results after conventional radiotherapy. ${ }^{5,6}$ SBRT has also been shown to decrease patient opioid use. ' Local control rates have ranged from $72.3 \%$ to $90.3 \%$ at 1 year, but there are significant differences in local control and overall survival rates based on primary tumor histology. ${ }^{8-12}$

A phase I/II study by Chang et al. considered the patterns of failure after spinal SBRT, and they identified the adjacent bone and the epidural space as the two most common areas for local failure. ${ }^{13}$ The presence of epidural disease prior to the start of treatment presents technical challenges regarding dose delivery to the epidural space, as sparing the spinal cord is imperative to preventing radiation myelopathy. ${ }^{14-16}$ Resection has been implemented in some such situations, demonstrating improved local control rates with surgical reduction in the epidural involvement. ${ }^{17,18}$ Other attempts to improve the efficacy of therapy have involved the implementation of concurrent, targeted molecular therapy or delivery of a higher radiation dose. ${ }^{19,20}$ Koyfman et al. also noted that a lower dose was associated with higher rates of marginal recurrence, and they found the presence of paraspinal disease to be associated with this outcome. ${ }^{21}$

Predictors for pain response are similar to those of local control. Again, primary tumor histology is predictive of clinical outcome, and a higher radiation dose and standardized uptake value on PET imaging result in decreased pain response. ${ }^{22-24}$ Toxicity rates are quite low after treatment, with particular concern applied to minimizing rates of vertebral compression fractures and radiation myelopathy. ${ }^{25-27}$ The safety of this technique allows for reirradiation, which provides impressive local control and pain response, often on the order of primary treatment. ${ }^{28-31}$

Approximately $50 \%$ of bone metastases occur in the spinal cord, with $60 \%-80 \%$ in the thoracic spine, $15 \%-$ $30 \%$ in the lumbar spine, less than $10 \%$ in the cervical spine, and $1 \%-7 \%$ in the sacral spine. ${ }^{32,33}$ There is a paucity of surgical and SBRT studies dedicated to the sacral spine, as it is the least common site for metastatic spinal spread. Notably, two surgical studies pointed toward improved outcomes in patients with surgical intervention or surgery followed by adjuvant radiotherapy. ${ }^{34,35}$ Only one dedicated study specifically considering the outcomes of cervical and sacral metastases after SBRT was identified, and it pointed toward improved local control in the cervical cohort and decreased local control in the sacral patients. ${ }^{36}$

The purpose of this study was to consider a large, single-institution cohort and investigate predictive factors for local control, local progression-free survival (LPFS), overall survival, and pain response. In particular, this analysis aims to consider potential differences in treatment outcomes between the different spinal regions using machine learning techniques, Cox proportional hazards analysis, and the Kaplan-Meier method.

\section{Methods}

\section{Inclusion and Exclusion Criteria}

All patients treated with SBRT for spinal metastases from August 2007 to November 2017 at one institution were tabulated and considered for retrospective review.
Patients with a tumor metastatic to the spine were included, while patients with benign tumors or primary spinal cord cancers were excluded. All cervical and sacral spine patients treated during this time period were included (due to the relative rarities of these two sites for metastatic disease), and a large set of thoracic and lumbar patients were included from a database previously exempted by the institutional review board. This database consisted of SBRT treatments with up to 5 fractions targeting tumors metastatic to the thoracic and lumbar spines. Exclusion criteria included a lack of clinical follow-up or a nonmetastatic tumor. The present study was also exempt by the institutional review board.

\section{Patient Characteristics}

Patient characteristics were tabulated to assess the following general characteristics: overall health, primary tumor, disease burden, spinal location, spinal disease details, and prior treatment. Overall health was assessed via age and Karnofsky Performance Status (KPS). The primary disease site and the corresponding histology were recorded. Overall disease burden was delineated as solitary metastasis or the presence of additional bone or visceral metastases. The spinal location was described as cervical, thoracic, lumbar, or sacral. Instances in which disease spanned two neuroanatomical regions were included in the analysis. Three cervicothoracic junction tumors were included in the cervical group, 1 thoracolumbar junction treatment was added to the thoracic subset, and 7 lumbosacral junction cases were incorporated into the sacral cohort. We felt that the treatment plans for tumors at the junctions were most similar to those in the groups selected. Disease details included the number of vertebrae involved, the stability of the patient's spine, and epidural or paraspinal tumor involvement. Finally, prior treatment (chemotherapy, surgery, or radiotherapy at that spinal level) was noted, including the dose, fractionation, and time from completion of any prior radiotherapy.

\section{Radiation Therapy}

Patient immobilization was undertaken with a fullbody vacuum bag system in order to provide stabilization and consistency for the planning CT scan. The dose was prescribed to the planning target volume (PTV), which was defined as the gross tumor volume plus $3-5 \mathrm{~mm}$ of margin, excluding the spinal canal. Intensity-modulated radiotherapy and volumetric-modulated arc therapy were considered, but a 3D conformal treatment planning approach was generally used. Coplanar gantry angles allowed for the preservation of the superior and inferior dosimetric borders. Typically, multiple coplanar static gantry delivery was utilized. In rare cases, noncoplanar static gantry was used, generally in treatments involving large volumes or particularly extensive disease. SBRT delivery utilized a 6-MV photon beam on a linear accelerator with a 2.5- to 4-mm-width multileaf collimator. On-board cone-beam CT was used prior to treatment and repeated several times (generally 2-4 times) during treatment to reassess patient positioning. A 6D robotic couch assisted in the alignment of the patient and localization of the tar- 
get to the planning CT. For planning purposes, the spinal cord organ at risk was defined as the spinal canal. A safety margin of $2 \mathrm{~mm}$ was applied to the cord for each treatment. Providers sought to minimize the dose prescribed to the spinal canal, with a maximum of 2 Gy per fraction less than the prescribed dose. For instance, in a course of therapy consisting of 24 Gy delivered in 3 fractions, the maximum tolerated dose to the spinal canal would have been 6 Gy per fraction.

\section{Study Endpoints}

This study was designed to assess overall survival, local control, and LPFS. Various pain outcomes were included as secondary endpoints, including pain status at the last clinical follow-up. The need for subsequent surgery or SBRT was also recorded. Toxicity was reported following the guidelines in the CTCAE (Common Terminology Criteria for Adverse Events) version 4.0. Two key toxicities included new or progressive fractures of treated vertebrae, which were assessed via imaging follow-up. This primarily consisted of CT or MRI every 2-3 months after completion of SBRT. Imaging was also utilized in order to assess for local failure. Clinical follow-up included pain (using the visual analog scale) and neurological assessments. Pain was tabulated as improved, stable, progressive, or no pain prior to and after SBRT. This same delineation was used regarding a patient's neurological symptoms. Finally, pain at the last clinical follow-up was recorded as pain free, mild/moderate, or severe.

\section{Initial Statistical Methods}

Cox proportional hazards analysis, univariate analysis, and the Kaplan-Meier method were used to more carefully assess the patient set. A threshold $p$ value of 0.05 was used to determine statistical significance. The Cox proportional hazards analysis was initially conducted with the entire set of patient characteristics to assess for potential predictive factors. Redundant variables (e.g., one-level spinal disease and multilevel disease) were trimmed to only one such variable to reduce covariance. Similarly, dose and fractions were not included, due to the high levels of covariance with biologically effective dose (BED). The BED was calculated using the following formula: $\mathrm{BED}=$ total dose $\times[1+$ dose per fraction/(alpha/beta ratio) $]$, where an alpha/beta ratio of $10 \mathrm{~Gy}$ was used. Univariate analyses were primarily conducted to analyze binary outcomes, including pain-free status at last follow-up, severe pain at last follow-up, or pain improvement after SBRT. The Kaplan-Meier method was utilized to assess time-dependent variables, such as local control, overall survival, and LPFS. These outcomes were substratified based on predictive factors determined by other aspects of the analysis, and $\mathrm{p}$ values were reported using a univariable log-rank test.

\section{Decision-Tree Analysis}

Decision-tree analysis was utilized in order to assess for predictive factors for two binary variables: local failure and pain-free status at last clinical follow-up. To do so, a Python script was written for analyzing the data, us- ing a range of statistical packages. These included pandas, matplotlib, lifelines, numpy, and sklearn. The ExtraTreesClassifier was used from sklearn to carry out the feature importance analysis, and the DecisionTreeClassifier was utilized for carrying out the decision-tree analysis. First, a feature importance analysis was undertaken. A figure was produced, and values corresponding to each independent variable's relative importance in predicting the dependent variable were determined. Independent variables were then eliminated from further analysis if they failed to reach the threshold value of 0.025 . A correlation heat map was generated to check for high covariance among the independent variables and allow for subsequent elimination of variables. Next, the data were split into training $(60 \%)$, test (25\%), and validation (15\%) sets. A decision-tree analysis was run through a loop considering a range of split, leaf, and maximum depth criteria, as well as considering a range of feature and selection criteria. This loop was run 20 consecutive times, and the model with the highest scores in accurately predicting the validation and test data subsets was selected.

\section{Results}

\section{Patients, Disease Details, and Treatment}

A total of 165 patients with 190 distinct lesions met all inclusion criteria for the study. The median patient age was 67.98 years, and the median KPS was 80 . Male patients accounted for $45 \%$ of the cohort, and female patients comprised $55 \%$. The most common primary tumor was breast (36\%), followed by non-small cell lung cancer (15\%) and prostate (11\%); $59 \%$ of lesions were diagnosed as adenocarcinoma. Patients presented at a median of 48.43 months after primary diagnosis, and, while $8 \%$ of treated tumors were the solitary sites of metastatic disease, $87 \%$ and $37 \%$ of cases involved additional bone or visceral metastases, respectively (Table 1).

Lesions were distributed throughout the cervical (19\%), thoracic (43\%), lumbar (19\%), and sacral (18\%) spines. Disease involved multiple spinal levels in $47 \%$ of cases, and $15 \%$ presented for SBRT with a compression fracture. Nonetheless, $87 \%$ of treatments involved spinal stability at the time of SBRT. Epidural and paraspinal involvement were noted in $34 \%$ and $27 \%$ of lesions, respectively. While $86 \%$ of treatments involved prior chemotherapy, 39\% had prior radiotherapy (with a median 30 Gy delivered in 10 fractions 5.86 months prior to SBRT); 9\% had prior surgical intervention.

The median SBRT was 24 Gy delivered in a median of 3 fractions, equating to a median of $43.2 \mathrm{~Gy}$ in BED. Most patients received 3 fractions of SBRT (76\%), with 4 fractions being used in $17 \%$ of cases and 1,2, or 5 fractions each used in $1 \%-3 \%$ of cases. The most common treatment regimen was $24 \mathrm{~Gy}$ in 3 fractions (44\%). The maximum point dose per fraction to the spinal canal was a median of $6.63 \mathrm{~Gy}$, and the median PTV was $34.3 \mathrm{~cm}^{3}$. The following values were recorded for cervical, thoracolumbar, and sacral locations, respectively: median volume receiving $80 \%$ of the prescribed dose $(97 \%, 97 \%$, and $100 \%$ ), median volume receiving $90 \%$ of the prescribed dose $(94 \%, 95.5 \%$, and $100 \%)$, median dose delivered to 
TABLE 1. Patient demographics and radiotherapy characteristics

\begin{tabular}{|c|c|}
\hline & Value \\
\hline No. of patients & 165 \\
\hline No. of lesions & 190 \\
\hline Median age, yrs & 67.98 \\
\hline Male* & $75(45)$ \\
\hline Female* & $90(55)$ \\
\hline Median KPS score & 80 \\
\hline \multicolumn{2}{|l|}{ Primary tumor } \\
\hline Breast & $69(36)$ \\
\hline NSCLC & $29(15)$ \\
\hline Prostate & $21(11)$ \\
\hline Other & $71(37)$ \\
\hline Adenocarcinoma & $113(59)$ \\
\hline Other histology & $77(41)$ \\
\hline Median time since diagnosis, mos & 48.43 \\
\hline Solitary metastasis & $15(8)$ \\
\hline Additional bone metastases & $165(87)$ \\
\hline Visceral metastases & $71(37)$ \\
\hline Cervical spine & $37(19)$ \\
\hline Thoracic spine & $81(43)$ \\
\hline Lumbar spine & $37(19)$ \\
\hline Sacral spine & $35(18)$ \\
\hline Median dose, Gy & 24 \\
\hline Median no. of fractions & 3 \\
\hline Median BED, Gy & 43.2 \\
\hline Maximum point dose per fraction to spinal canal, Gy & 6.63 \\
\hline Median PTV, $\mathrm{cm}^{3}$ & 34.3 \\
\hline Median days btwn fractions & 4.67 \\
\hline \multicolumn{2}{|l|}{ No. of vertebrae involved } \\
\hline 1 & $101(53)$ \\
\hline 2 & $82(43)$ \\
\hline 3 & $7(4)$ \\
\hline Compression fracture & $29(15)$ \\
\hline Stability at time of SBRT & $166(87)$ \\
\hline Epidural involvement & $65(34)$ \\
\hline Paraspinal involvement & $51(27)$ \\
\hline Prior chemotherapy & $163(86)$ \\
\hline Prior surgery & $17(9)$ \\
\hline Prior radiotherapy at spinal level & $75(39)$ \\
\hline Median dose, Gy & 30 \\
\hline Median fraction & 10 \\
\hline Median time from prior radiotherapy, mos & 5.86 \\
\hline
\end{tabular}

Values represent the number of lesions (\%) unless stated otherwise.

${ }^{*}$ Values represent the number of patients (\%).

$80 \%$ of the volume $(6.9,8.8$, and $7.4 \mathrm{~Gy} /$ fraction), and median dose delivered to $90 \%$ of the volume $(6.1,7.8$, and $7.2 \mathrm{~Gy} /$ fraction). Patients had a median 4.67 days between fractions. This somewhat longer time than at other centers is largely because many patients were treated with onceweekly fractionation.
TABLE 2. Patient outcomes

\begin{tabular}{|c|c|}
\hline & Value \\
\hline 24-mo local control & $80 \%$ \\
\hline Insufficient follow-up to determine & $13(7)$ \\
\hline Median overall survival, mos & 13.67 \\
\hline Patients deceased & $105(64)$ \\
\hline Subsequent surgery & $6(3)$ \\
\hline Subsequent SBRT & $32(17)$ \\
\hline Duration of pain follow-up, mos & 12.62 \\
\hline Pain improved after SBRT & $137(72)$ \\
\hline Pain stable after SBRT & $27(14)$ \\
\hline Pain progressive after SBRT & $9(5)$ \\
\hline No pain before or after SBRT & $15(8)$ \\
\hline Pain free at last follow-up & $99(52)$ \\
\hline Severe pain at last follow-up & $21(11)$ \\
\hline \multicolumn{2}{|l|}{ Neurological symptoms after SBRT } \\
\hline Improved & $7(4)$ \\
\hline Stable & $29(15)$ \\
\hline Progressive & $21(11)$ \\
\hline None & $130(68)$ \\
\hline Median LPFS, mos & 12.12 \\
\hline \multicolumn{2}{|l|}{ Toxicity grade } \\
\hline 1 & $54(28)$ \\
\hline 2 & $6(3)$ \\
\hline$\geq 3$ & $0(0)$ \\
\hline New fracture of treated vertebrae & $3(2)$ \\
\hline Progressive fracture of treated vertebrae & $2(1)$ \\
\hline
\end{tabular}

Values represent the number of lesions (\%) unless stated otherwise.

\section{Outcomes}

In 13 cases, there was insufficient imaging follow-up to determine local control. Using the Kaplan-Meier method, an overall 24-month local control rate of $80 \%$ was determined. Patients had a median overall survival of 13.67 months and a median LPFS of 12.12 months. Patients in 6 cases underwent subsequent surgery, while patients in 32 cases had subsequent SBRT for local, regional, or distant failure (Table 2).

A median of 12.62 months of pain follow-up was obtained: $72 \%$ of patients had improvement of pain after SBRT, while $14 \%$ were stable and only $5 \%$ felt increased pain. Fifty-two percent of patients reported no pain at the last clinical follow-up, and $11 \%$ had severe pain at the last follow-up. The majority of patients $(68 \%)$ had no neurological symptoms before or after SBRT.

Reported toxicity was quite low in this analysis; 54 treatments $(28 \%)$ resulted in grade 1 toxicity, and only 6 instances of grade 2 toxicity were reported. The most common toxicities included fatigue, dysphagia, and radiationinduced dermatitis, with 23, 14, and 13 cases, respectively. There were no observed grade $\geq 3$ side effects. Notably, there were only 3 new fractures and 2 progressive fractures of treated vertebrae. No radiation myelopathy was observed. 
TABLE 3. Key $p$ values from univariate and Kaplan-Meier analyses concerning the spinal regions

\begin{tabular}{lccc}
\hline \multicolumn{1}{c}{ Endpoint } & Sacral vs Thoracolumbar & Sacral vs Cervical & Thoracolumbar vs Cervical \\
\hline Epidural involvement & 0.34 & 0.52 & 0.86 \\
\hline Paraspinal involvement & 0.16 & 0.13 & 0.66 \\
\hline PTV & 0.27 & $2.53 \times 10^{-3^{*}}$ & $5.87 \times 10^{-8^{*}}$ \\
\hline PTV $>50 \mathrm{~cm}^{3}$ & 0.14 & $3.93 \times 10^{-3^{*}}$ & $8.76 \times 10^{-3^{*}}$ \\
\hline Kaplan-Meier analysis & & & \\
\hline Local failure & $0.01^{*}$ & $<0.005^{*}$ & 0.21 \\
\hline LPFS & $<0.005^{*}$ & $<0.005^{*}$ & 0.17 \\
\hline Local failure & $0.029^{*}$ & $0.0028^{*}$ & 0.11 \\
\hline Pain free at last follow-up & $0.0027^{*}$ & $0.0001^{*}$ & 0.078 \\
\hline Severe pain at last follow-up & 0.11 & $0.043^{*}$ & 0.38 \\
\hline * Met statistical significance & & &
\end{tabular}

${ }^{*}$ Met statistical significance.

\section{Prognostic Factors}

Initial stratifications of the data indicated some differences in outcomes based on the spinal region of the treated tumor. For instance, $70 \%$ of cervical spine treatments resulted in pain-free status, compared with $57 \%, 49 \%$, and $27 \%$ of thoracic, lumbar, and sacral treatments, respectively. Patients with thoracic and lumbar lesions had outcomes similar to the cohort as a whole, but patients with cervical and sacral spine lesions often had different values. Compared with the overall local failure rate of $15 \%$, patients with cervical spine lesions only had $6 \%$ local failure, and patients with sacral pathology showed a rate of $34 \%$. The 24-month local control of thoracolumbar lesions was $87 \%$; however, the 36-month local control rates via the KaplanMeier method were $86 \%, 73 \%$, and $44 \%$ for the cervical, thoracolumbar, and sacral treatments, respectively. Comparably, the 36-month LPFS rates were $85 \%, 67 \%$, and $35 \%$, respectively. Similarly, sacral lesions resulted in either no pain or pain improvement in only $60 \%$ of instances, compared with $86 \%$ in cervical spine patients. Univariate analysis demonstrated decreased rates of local control ( $p$ $=0.0028)$ and pain-free status $(p=0.0001)$ but increased rates of severe pain $(\mathrm{p}=0.043)$ in sacral lesions compared with cervical lesions. Sacral lesions also had increased local failure $(\mathrm{p}=0.029)$ and decreased pain-free status $(\mathrm{p}=0.0027)$ compared with thoracolumbar lesions, with a trend toward increased rates of severe pain $(\mathrm{p}=0.11)$. Finally, cervical spine patients trended toward improved rates of local control $(\mathrm{p}=0.11)$ and pain-free status $(\mathrm{p}=$ 0.078 ) compared with thoracolumbar patients (Table 3 ).

Cox proportional hazards analysis was implemented to assess for other potential predictive factors (Fig. 1). Prior chemotherapy was predictive of decreased overall survival (HR 6.88, 95\% CI 1.37-34.62). Paraspinal involvement also trended toward decreased overall survival $(\mathrm{p}=$ 0.06 ). Breast primary tumor was predictive of decreased local failure (HR 0.10, 95\% CI 0.02-0.56), while female sex demonstrated increased local failure (HR 4.98, 95\% CI 1.06-23.34).

The Kaplan-Meier method was used to demonstrate overall survival and local control for the entire cohort (Fig. 2). Paraspinal disease $(\mathrm{p}=0.01)$ and prior chemotherapy $(\mathrm{p}=0.01)$ were predictive of decreased overall survival on Kaplan-Meier analysis (Supplemental Fig. 1). Additionally, disease with a primary breast malignancy showed improved local control $(\mathrm{p}<0.005)$ and LPFS ( $\mathrm{p}=$ 0.01 ) (Fig. 3). Stratification by spinal region was illustrated for local failure and LPFS (Fig. 4). The sacral spine lesions demonstrated decreased local control $(\mathrm{p}=0.01)$ and LPFS $(p<0.005)$ compared with those of the thoracolumbar spine. The cervical spine cases demonstrated improved local control $(\mathrm{p}<0.005)$ and LPFS $(\mathrm{p}<0.005)$ compared with the sacral spine cases and trended toward improved local control $(p=0.21)$ and LPFS $(p=0.17)$ relative to the thoracolumbar spine.

When the spinal region subsets were considered, epidural involvement was not predictive of local failure either in the entire cohort or within the sacral cohort itself. Additionally, although patients with sacral pathology had an increase in epidural and paraspinal involvement relative to the rest of the cohort (43\% and 40\% compared with $32 \%$ and $24 \%$, respectively), these differences were not statistically significant. Although PTV itself was not predictive of local failure, PTV $>50 \mathrm{~cm}^{3}$ was predictive of local failure $(p=0.04)$. Furthermore, cervical spine cases were less likely to have disease with a PTV of $>50 \mathrm{~cm}^{3}$ compared with the thoracolumbar $\left(\mathrm{p}=5.87 \times 10^{-8}\right)$ and sacral $(\mathrm{p}$ $\left.=3.9 \times 10^{-3}\right)$ regions. Figure 5 shows that Kaplan-Meier analysis demonstrated trends toward improvement of local control and LPFS with a PTV of $\leq 50 \mathrm{~cm}^{3}$.

\section{Decision-Tree Analysis}

Decision-tree analysis was implemented to develop models for pain-free status at last follow-up and local failure. Feature importance testing for pain-free status resulted in the elimination of prostate primary, solitary metastasis, additional bone metastases, prior chemotherapy, prior surgery, and stability at the time of SBRT. The highest-fidelity model predicted the validation set in $90 \%$ of instances and the test set in $84.1 \%$ of cases, and the first split in the decision tree was cervical location (Supplemental Fig. 2). Feature importance testing for local failure similarly eliminated prostate primary, solitary metastasis, additional bone metastases, prior chemotherapy, prior surgery, thoracolumbar spine, and stability at the time of SBRT. The highest-fidelity resulting model predicted the 

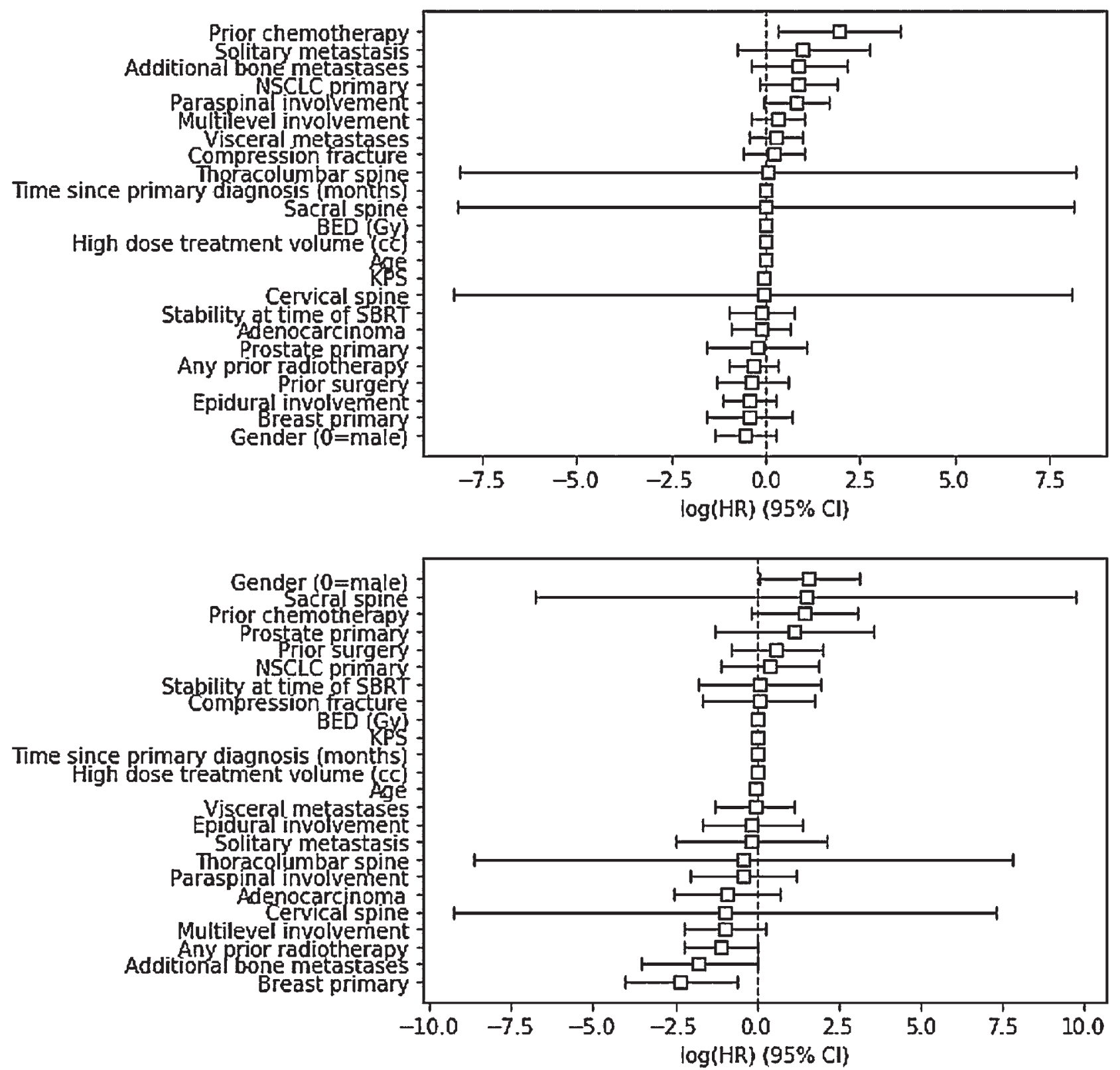

FIG. 1. Cox proportional hazards analysis for overall survival (upper) and local failure (lower). NSCLC = non-small cell lung cancer.

validation set in $94.74 \%$ of instances and the test set in $78.57 \%$ of cases, and the first split in the decision tree was sacral location (Supplemental Fig. 3).

\section{Discussion}

The principal result of this study is the local control risk stratification by spinal region, as demonstrated in Fig. 4. To our knowledge, this is the first such delineation in the literature involving the cervical, thoracolumbar, and sacral spines. The primary justification for this distinction is the result of the Kaplan-Meier analysis demonstrating improved local control and LPFS for cervical lesions, relatively expected outcomes for thoracolumbar treatment, and decreased local control and LPFS for sacral cases. A similar relationship was demonstrated on univariate analysis regarding the secondary endpoints of pain-free status at last follow-up and severe pain at last follow-up.

Additional support for this conclusion was provided by the decision-tree analysis for local failure and pain-free status. The first split in the highest-fidelity model for local failure was sacral location, and the first split in the painfree status model was cervical location. There was high variance between the different models produced through this method of machine learning, as would be expected for a relatively small data set compared with other "big data" analyses; however, in both instances, a model was produced that predicted the desired outcome with reason- 


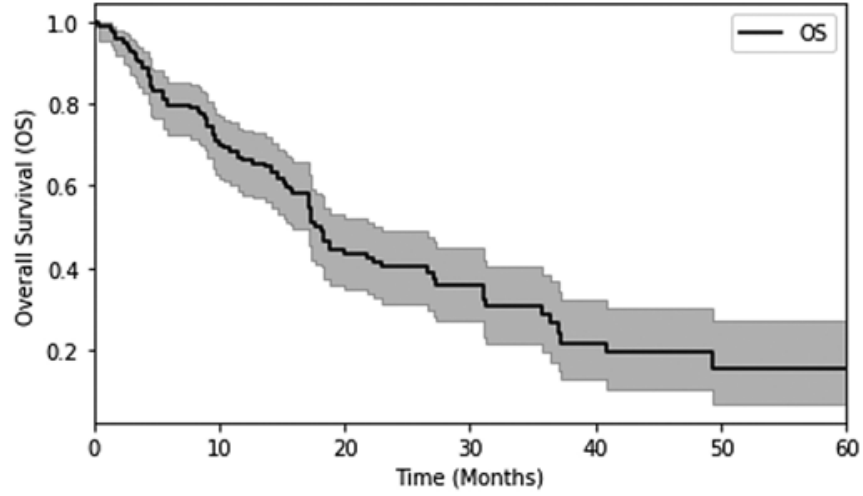

At risk

OS 165

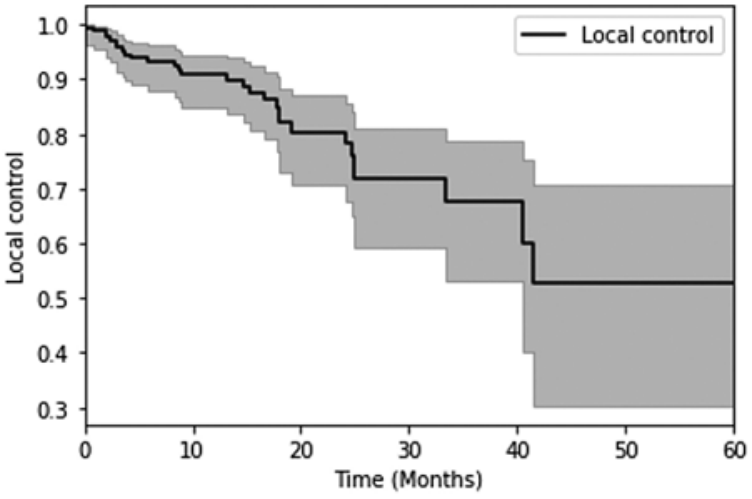

At risk

FIG. 2. Overall survival (left) and local control (right) for the entire cohort. Gray areas indicate the $95 \% \mathrm{Cls}$.

able accuracy. Coupled with the feature importance analysis (through which thoracolumbar location was eliminated from the local failure model), these results suggest that tumor location in the cervical or sacral region has a significant prognostic role for local control and pain response.

The most similar study to the present analysis directly compared SBRT for cervical and sacral lesions, demonstrating improved local control at 1 year and 2 years after the cervical spine treatments. ${ }^{36}$ The authors proposed four chief reasons to explain these results. First, their cohort demonstrated increased rates of radioresistant histologies in the sacral cohort. While this finding was present in their data, this data set found no such difference. Second, they commented on increased rates of epidural disease in patients with sacral lesions and the absence of epidural disease in the sacral cohort predicted for improved local control. The present study also noted an increase in epidural disease in the sacral spine patients, but its absence was not predictive of any outcome. Furthermore, the increase in epidural disease in patients with sacral lesions was not statistically significant. Third, they discussed the technical challenges regarding treatment of the sacral spine with SBRT, citing the lack of specific clinical tumor volume contouring guidelines and contouring of the thecal sac as opposed to individual nerve roots. We agree that these anatomical considerations are significant, and they present further challenges to multiinstitutional studies, due to the diversity in treatment planning and delivery.

Zeng et al. also reported that clinical tumor volumes and PTVs in sacral lesions were approximately 50\% greater than in cervical cases. ${ }^{36}$ At least two studies have commented on the relationship between tumor volume and local failure in the setting of SBRT for spine metastases, and a threshold of PTV $>50 \mathrm{~cm}^{3}$ was identified. ${ }^{8,37}$ This threshold is not commonly utilized, however, and many other studies have not demonstrated a relationship between PTV and local control., ${ }^{9,36}$ Even so, Chang et al. advocated for more liberal spinal cord dose constraints for patients without prior radiotherapy in an effort to reduce failures in the epidural space. ${ }^{13}$ We hypothesize that this statement is also true for patients treated for sacral spine metastases, as the technical challenges previously discussed may also increase the risk for local progression in the epidural space. Local control rates may also be improved with surgical intervention prior to radiotherapy, which can downgrade epidural disease. ${ }^{17,18,34,35}$ Even minimally invasive surgery in patients without spine instability, such as sacroplasty, may be of benefit. ${ }^{38}$ Sacral tumors are often located close
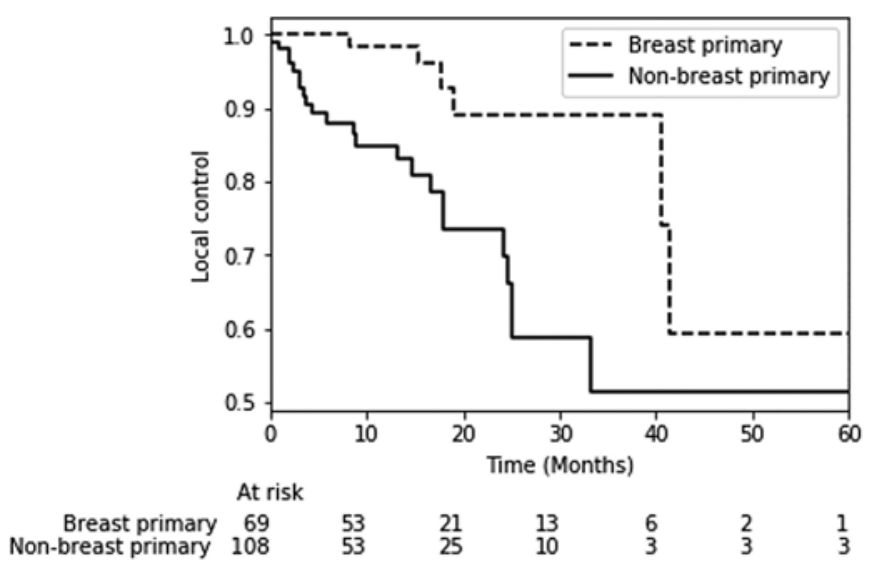

FIG. 3. Lesions with a breast primary tumor have improved local control (left) and LPFS (right).

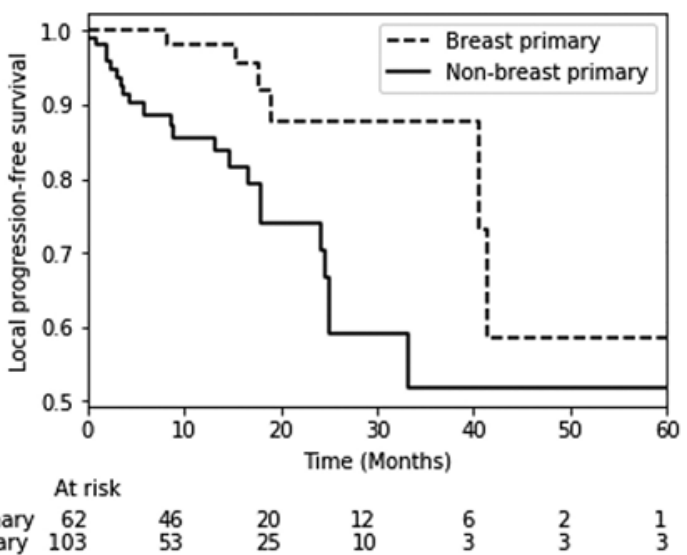



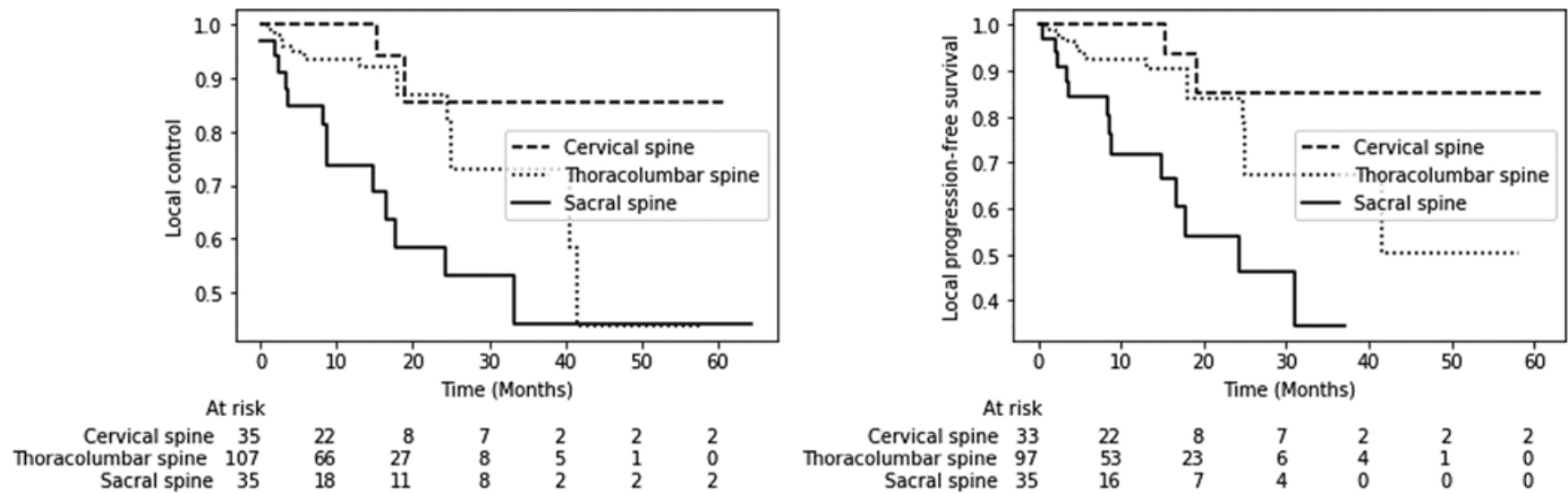

FIG. 4. Stratifications by spinal level (cervical, thoracolumbar, and sacral) are shown for local control (left) and LPFS (right).

to major neural structures and involve complex anatomy, so establishing multidisciplinary care to individualize patient treatment plans is vital for optimizing outcomes. ${ }^{39}$

The moderately improved outcomes in the cervical spine cohort are a novel finding compared with the existing literature. In addition to improved local control and LPFS, cervical spine treatments were less likely to require subsequent SBRT $\left(\mathrm{p}=2.48 \times 10^{-9}\right)$. One potential explanation would involve the decreased rate of PTV $>50 \mathrm{~cm}^{3}$ in the cervical cohort compared with the thoracolumbar and sacral subsets. Additionally, the cervical spine treatments included a higher fraction of primary breast tumors $(46 \%)$ than the rest of the cohort, which may have contributed to the improved outcomes.

Other predictive factors were identified during the analysis, and they serve to support previous observations in the literature. Prior chemotherapy portended decreased overall survival, which is consistent with systemic disease status playing a dominant role in predicting overall survival in metastatic cancer patients. ${ }^{11}$ Primary breast tumors demonstrated improved local control and LPFS, which is consistent with previous literature illustrating the impact of tumor histology. ${ }^{8-10,12}$ Finally, paraspinal disease was predictive of decreased overall survival on Kaplan-Meier analysis. This result differs from previous analyses, which generally only found an association with marginal recurrence. ${ }^{21}$ In this case, paraspinal involvement was predictive of PTV $>50 \mathrm{~cm}^{3}(\mathrm{p}=0.025)$, which may also serve to partly explain its prognostic value.

There are four chief limitations of this study. First, the Bilsky score was not recorded, which would have further classified the epidural involvement of disease. Grading of the epidural disease (or at least a distinction between highgrade and low-grade involvement) could have been beneficial since it is possible that high-grade epidural disease portends a distinct disease outcome, compared with overall epidural involvement. Second, SINS (Spinal Instability Neoplastic Score) would have better described spinal instability, compared with a binary assessment. Third, opioid use was not recorded to further detail the pain response to treatment. This additional information would have allowed us to further stratify whether opioid use changed after SBRT and whether or not this might have impacted pain-free status at last follow-up. Finally, the relatively few sacral and cervical spine treatments included in this study did not allow for the application of more rigorous machine learning techniques without the significant risk of overfitting the data. A multiinstitutional study is highly recommended to compile large patient sets of cervical and sacral spinal metastases.
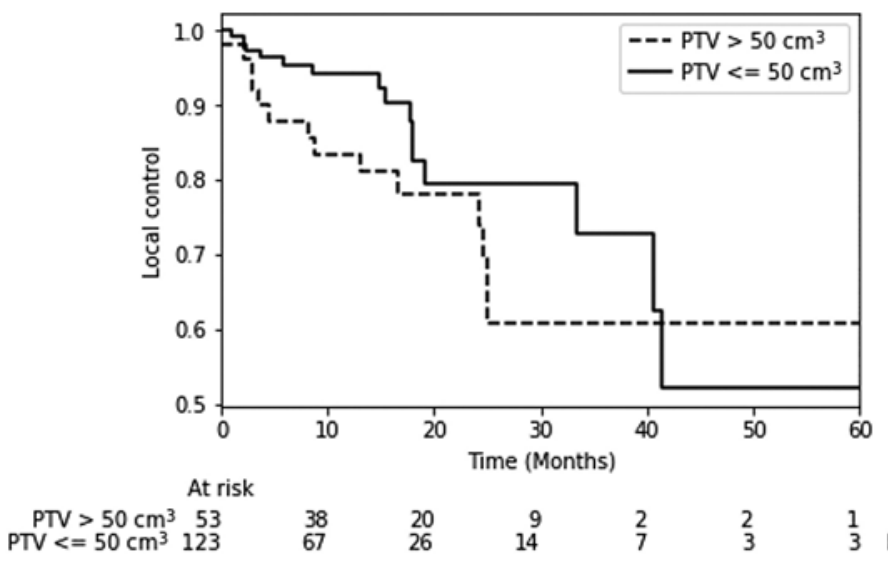

At risk
$\frac{1}{3}$ PTV $>50 \mathrm{~cm}^{3} 43$
PTV $<=50 \mathrm{~cm}^{3} 109$

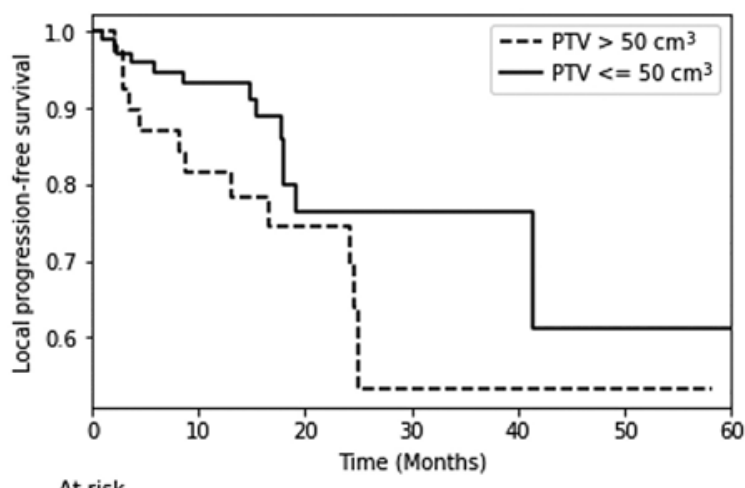

PTV $<=50 \mathrm{~cm}^{3} 123$

FIG. 5. The Kaplan-Meier method was used to stratify local control $(p=0.14)$ (left) and LPFS $(p=0.08)$ (right) by PTV $>50 \mathrm{~cm}^{3}$. 


\section{Conclusions}

This study presents a novel risk stratification for local failure and LPFS by spinal region. Patients with sacral spine lesions may have decreased local control due to increased PTV, especially with a PTV $>50 \mathrm{~cm}^{3}$. Multidisciplinary care should be emphasized in these patients, and both surgical intervention and radiotherapy should be strongly considered.

\section{References}

1. Sangha A, Korol R, Sahgal A. Stereotactic body radiotherapy for the treatment of spinal metastases: an overview of the University of Toronto, Sunnybrook Health Sciences Odette Cancer Centre, Technique. J Med Imaging Radiat Sci. 2013; 44(3):126-133.

2. Pan H, Simpson DR, Mell LK, et al. A survey of stereotactic body radiotherapy use in the United States. Cancer. 2011; 117(19):4566-4572.

3. Alongi F, Arcangeli S, Filippi AR, et al. Review and uses of stereotactic body radiation therapy for oligometastases. Oncologist. 2012;17(8):1100-1107.

4. Spencer KL, van der Velden JM, Wong E, et al. Systematic review of the role of stereotactic radiotherapy for bone metastases. J Natl Cancer Inst. 2019;111(10):1023-1032.

5. Sprave T, Verma V, Förster R, et al. Randomized phase II trial evaluating pain response in patients with spinal metastases following stereotactic body radiotherapy versus threedimensional conformal radiotherapy. Radiother Oncol. 2018; 128(2):274-282.

6. Guckenberger M, Sweeney RA, Hawkins M, et al. Doseintensified hypofractionated stereotactic body radiation therapy for painful spinal metastases: results of a phase 2 study. Cancer. 2018;124(9):2001-2009.

7. Wang XS, Rhines LD, Shiu AS, et al. Stereotactic body radiation therapy for management of spinal metastases in patients without spinal cord compression: a phase 1-2 trial. Lancet Oncol. 2012;13(4):395-402.

8. Mehta N, Zavitsanos PJ, Moldovan K, et al. Local failure and vertebral body fracture risk using multifraction stereotactic body radiation therapy for spine metastases. Adv Radiat Oncol. 2018;3(3):245-251.

9. Tseng CL, Soliman H, Myrehaug S, et al. Imaging-based outcomes for 24 Gy in 2 daily fractions for patients with de novo spinal metastases treated with spine stereotactic body radiation therapy (SBRT). Int J Radiat Oncol Biol Phys. 2018; 102(3):499-507.

10. Ito K, Ogawa H, Shimizuguchi T, et al. Stereotactic body radiotherapy for spinal metastases: clinical experience in 134 cases from a single Japanese institution. Technol Cancer Res Treat. 2018;17:1533033818806472.

11. Wang CJ, Christie A, Lin MH, et al. Safety and efficacy of stereotactic ablative radiation therapy for renal cell carcinoma extracranial metastases. Int J Radiat Oncol Biol Phys. 2017;98(1):91-100.

12. Chao ST, Koyfman SA, Woody N, et al. Recursive partitioning analysis index is predictive for overall survival in patients undergoing spine stereotactic body radiation therapy for spinal metastases. Int J Radiat Oncol Biol Phys. 2012;82(5): $1738-1743$

13. Chang EL, Shiu AS, Mendel E, et al. Phase I/II study of stereotactic body radiotherapy for spinal metastasis and its pattern of failure. J Neurosurg Spine. 2007;7(2):151-160.

14. Cox BW, Spratt DE, Lovelock M, et al. International Spine Radiosurgery Consortium consensus guidelines for target volume definition in spinal stereotactic radiosurgery. Int $J$ Radiat Oncol Biol Phys. 2012;83(5):e597-e605.

15. Balagamwala EH, Cherian S, Angelov L, et al. Stereotactic body radiotherapy for the treatment of spinal metastases. $J$ Radiat Oncol. 2012;1(3):255-265.

16. Garg AK, Wang XS, Shiu AS, et al. Prospective evaluation of spinal reirradiation by using stereotactic body radiation therapy: The University of Texas MD Anderson Cancer Center experience. Cancer. 2011;117(15):3509-3516.

17. Al-Omair A, Masucci L, Masson-Cote L, et al. Surgical resection of epidural disease improves local control following postoperative spine stereotactic body radiotherapy. Neuro Oncol. 2013;15(10):1413-1419.

18. Tseng CL, Eppinga W, Charest-Morin R, et al. Spine stereotactic body radiotherapy: indications, outcomes, and points of caution. Global Spine J. 2017;7(2):179-197.

19. Park S, Kim KH, Rhee WJ, et al. Treatment outcome of radiation therapy and concurrent targeted molecular therapy in spinal metastasis from renal cell carcinoma. Radiat Oncol $J$. 2016;34(2):128-134.

20. Yamada Y, Bilsky MH, Lovelock DM, et al. High-dose, single-fraction image-guided intensity-modulated radiotherapy for metastatic spinal lesions. Int J Radiat Oncol Biol Phys. 2008;71(2):484-490.

21. Koyfman SA, Djemil T, Burdick MJ, et al. Marginal recurrence requiring salvage radiotherapy after stereotactic body radiotherapy for spinal metastases. Int J Radiat Oncol Biol Phys. 2012;83(1):297-302.

22. Miller JA, Balagamwala EH, Chao ST, et al. Spine stereotactic radiosurgery for the treatment of multiple myeloma. $J$ Neurosurg Spine. 2017;26(3):282-290.

23. Lee E, Kim TG, Park HC, et al. Clinical outcomes of stereotactic body radiotherapy for spinal metastases from hepatocellular carcinoma. Radiat Oncol J. 2015;33(3):217-225.

24. Adli M, Kuzhan A, Alkis H, et al. FDG PET uptake as a predictor of pain response in palliative radiation therapy in patients with bone metastasis. Radiology. 2013;269(3):850-856.

25. Chang JH, Shin JH, Yamada YJ, et al. Stereotactic body radiotherapy for spinal metastases: What are the risks and how do we minimize them? Spine (Phila Pa 1976). 2016;41(suppl 20):S238-S245.

26. Wong CC, McGirt MJ. Vertebral compression fractures: a review of current management and multimodal therapy. $J$ Multidiscip Healthc. 2013;6:205-214.

27. Alexandru D, So W. Evaluation and management of vertebral compression fractures. Perm J. 2012;16(4):46-51.

28. Hashmi A, Guckenberger M, Kersh R, et al. Re-irradiation stereotactic body radiotherapy for spinal metastases: a multiinstitutional outcome analysis. J Neurosurg Spine. 2016; 25(5):646-653.

29. Joaquim AF, Ghizoni E, Tedeschi H, et al. Stereotactic radiosurgery for spinal metastases: a literature review. Einstein (Sao Paulo). 2013;11(2):247-255.

30. Thibault I, Campbell M, Tseng CL, et al. Salvage stereotactic body radiotherapy (SBRT) following in-field failure of initial SBRT for spinal metastases. Int J Radiat Oncol Biol Phys. 2015;93(2):353-360.

31. Myrehaug S, Sahgal A, Hayashi M, et al. Reirradiation spine stereotactic body radiation therapy for spinal metastases: systematic review. J Neurosurg Spine. 2017;27(4):428-435.

32. Kim J, Seol Y, Jang HS, Kang YN. Spinal stereotactic body radiotherapy (SBRT) planning techniques. In: Osibote A, ed. Ionizing and Non-ionizing Radiation. IntechOpen; 2020. Accessed July 23, 2020. https://www.intechopen.com/books/ ionizing-and-non-ionizing-radiation/spinal-stereotactic-bodyradiotherapy-sbrt-planning-techniques

33. Mika A, Mesfin A. Update on the management of sacral metastases. JBJS Rev. 2018;6(7):e8.

34. Quraishi NA, Giannoulis KE, Edwards KL, Boszczyk BM. Management of metastatic sacral tumours. Eur Spine J. 2012; 21(10):1984-1993.

35. Du Z, Guo W, Yang R, et al. What is the value of surgical 
intervention for sacral metastases? PLoS One. 2016;11(12): $\mathrm{e} 0168313$.

36. Zeng KL, Myrehaug S, Soliman H, et al. Stereotactic body radiotherapy for spinal metastases at the extreme ends of the spine: imaging-based outcomes for cervical and sacral metastases. Neurosurgery. 2019;85(5):605-612.

37. Tao R, Bishop AJ, Brownlee Z, et al. Stereotactic body radiation therapy for spinal metastases in the postoperative setting: a secondary analysis of mature phase 1-2 trials. Int $J$ Radiat Oncol Biol Phys. 2016;95(5):1405-1413.

38. Masala S, Konda D, Massari F, Simonetti G. Sacroplasty and iliac osteoplasty under combined CT and fluoroscopic guidance. Spine (Phila Pa 1976). 2006;31(18):E667-E669.

39. Nader R, Rhines LD, Mendel E. Metastatic sacral tumors. Neurosurg Clin N Am. 2004;15(4):453-457.

\section{Disclosures}

The wife of Dr. Kowalchuk is a senior technical product manager at GE Healthcare.

\section{Author Contributions}

Conception and design: Kowalchuk, Sheehan, Kersh. Acquisition of data: Kowalchuk. Analysis and interpretation of data: Kowalchuk, Waters, Sheehan. Drafting the article: Kowalchuk, Spencer. Critically revising the article: all authors. Reviewed submitted version of manuscript: all authors. Approved the final version of the manuscript on behalf of all authors: Kowalchuk. Statistical analysis: Kowalchuk. Administrative/technical/material support: Richardson, Spencer, McAllister, Sheehan, Kersh. Study supervision: Richardson, Larner, McAllister, Sheehan, Kersh.

\section{Supplemental Information}

Online-Only Content

Supplemental material is available with the online version of the article.

Supplemental Figs. 1-3. https://thejns.org/doi/suppl/10. 3171/2020.6.SPINE20861.

\section{Correspondence}

Roman O. Kowalchuk: Riverside, Radiosurgery Center, Newport News, VA.roman.kowalchuk@rivhs.com; okowal17@gmail.com. 